abnormalities; but these are not described in either of the two articles. Had that information been provided two different patterns would have emerged. In one most of the pulmonary opacities would have been recognisably segmental or subsegmental, and evidence of bronchial plugs or casts might have been obtained from tomography or bronchoscopy, or from the subsequent development of proximal bronchiectasis. In the other most of the opacities would have been consistent with peripheral pulmonary infiltrates, and evidence of bronchial obstruction would have been absent. By failing to make this distinction the authors have not taken into account the very real possibility that patients with these two forms of allergic bronchopulmonary aspergillosis may differ in several relevant respects, including response to prednisolone and liability to sustain permanent bronchopulmonary damage in the form of distal bronchiectasis and fibrosis. Similarly, they did not choose to identify differences they knew to exist within their "non-aspergillosis" group, which might have influenced not only the response to prednisolone but also some of the other measures on which comparison of the two groups was based.

The authors have thus been comparing two crudely selected groups of patients withou first considering whether the undisclosed differences within each group could have invalidated the statistical computations used to evaluate differences between the two groups. No conclusions drawn from studies conducted along these lines can be wholly reliable.

IAN WB GRANT Burnwynd, West Lothian EH27 8EA

1 Chapman BJ, Capewell SD, Alexander F, Greening AP, Crompton GK. Pulmonary eosinophilia with and without allergic bronchopulmonary aspergillosis. Thorax 1989;44:919-4.

2 Capewell S, Chapman BJ, Alexander F, Greening AP, Crompton GK. Corticosteroid treatment and prognosis in pulmonary eosinophilia. Thorax 1989;44:925-9. are two physiologists, an anaesthetist and a professor of medicine. In the preface they state that the book is most suitable for medica and (non-medical) graduate students. This is a broad remit but, within medicine, I think that the book would be most useful for those studying anaesthetics or intensive care rather than respiratory medicine. It covers areas that are not usually covered in physiology textbooks, in particular the pulmonary and bronchial circulations, lung fluid balance, the use of ventilators, and the physiology of high altitude and diving medicine. I found these very welcome, and the section on the clinical use of pulmonary function test was also good I have some criticisms about the other sections. For example, in the discussion of ventilation the only spirometer mentioned was a water spirometer, a piece of equipment not generally found outside physiology laboratories. In the section on diffusion there was no mention of specific gas transfer. In the section on oxygen transport there was no mention of the relation of cardiac output to oxygen delivery. The chapter on ventilatory control had little on the sleep apnoea syndrome, Cheyne Stokes respiration, or exercise physiology. The drawings are clear and well presented. Photographs are mostly lifted from other texts but are no worse for that Each chapter is followed by a series of questions related to that chapter, which are mean to be problem solving within the subjects covered by the chapter but are actually calculations based on data presented in the chapter. Overall this is a well written and well presented book, which suffers slightly, I think, from a lack of direction. It is not clear whether it is meant for those studying physiology, those studying medicine, or those who graduated in medicine and are studying for specialties, such as anaesthetics or respiratory medicine, where a good knowledge of clinical respiratory physiology is essential. The book would be most useful for those interested in anaesthetics, because of the chapters on fluid balance and ventilators. As a respiratory physician I would have liked to see more on the relevance of the various aspects of clinical physiology to the common and less common diseases affecting the patients we see.-AP

Biopsy Pathology of the Pulmonary Vasculature. CA Wagenvoort, WJ Mool. (Pp 362; £59.50.) New York: Routledge, Chapman and Hall, 1989. ISBN 0-41227940-1.

This is the latest volume in the series currently published by Chapman and Hall Medical. These books are aimed primarily at the practising pathologist, and are intended to be used more as bench books than as definitive reference works. Professor Wagenvoort and Dr Mool are international authorities on the pathology of pulmonary vascular disease, and this book is in many ways an update of Pathology of Pulmonary Hypertension, which first appeared in 1977. Its emphasis, however, is oriented towards the open lung biopsy diagnosis of the whole spectrum of pulmonary vascular disease rather than hypertensive states alone. The first three chapters deal with the microanatomy of the normal pulmonary vascular bed, and with techniques for obtain- ing and processing biopsy specimens. Specific details of the changes in various disorders are covered in the ensuing chapters, which include sections on plexogenic pulmonary hypertension, hypoxic pulmonary vascular disease, and the vasculopathies associated with chronic congestive states and conditions in which blood flow and pulse pressure are diminished. Developmental disorders, tumours, and primary and reactive inflammatory disease of the pulmonary vascular bed are also described. The chapter dealing with the evaluation of preoperative biopsy specimens from patients with congenital heart disease is of particular value. This is an intensely practical book, based on conventional light microscopy and the standard staining methods available in every laboratory. On those grounds alone, it is essential reading for everyone concerned in the diagnosis and treatment of pulmonary vascular disease. But it is much more than just a glorified atlas. The authors have also succeeded in producing a standard reference text-and an outstanding example of how simple techniques allied to clear thinking can elucidate the most complex problems.CWE

\section{Allergy and Asthma-New Trends and} Approaches to Therapy. AB Kay. (Pp 277; £35.) Oxford: Blackwell, 1989. ISBN 0632 022396 .

This book describes current research into the development of drugs which, through modulating allergic responses, are potential treatments for asthma. It also intends to give the reader new ideas for future research. It contains a number of manuscripts gathered from a meeting in November 1987 and includes some of the audience discussion. About half of the contributors are from the pharmaceutical industry. The book is divided into three sections, the first describing lipid mediators and their antagonists, the second modulation of the specific immune response, and the third agents that suppress inflammation. Of the three sections, the first is the most successful, with good reviews of the potential for development of drugs inhibiting lipid mediators. I particularly enjoyed the chapter from Dr Philips, a clearly stated case that would act as a good model for discussing the inhibitors of any other mediators. The section on immunotherapy was the most disappointing and suffers from the time lapse between the meeting and publication of the book, and also from a rather uncritical assessment of immunotherapy by Dr Dreborg. Potentially the most exciting section on anti-inflammatory drugs confines itself to discussing nedocromil sodium, antihistamines, citirizine, azelatine, and the steroid derived peptide lipocortin. The chapters on nedocromil, lipocortin, and citirizine provide a balanced review of inflammation available at the time of writing. In the chapter on azelatine, however, the authors allow themselves some rather uncricital speculation about the clinical benefit of the drug. Overall the book would be of value for obtaining some background information on the subject for those starting out. Its lack of up to date information on rapidly changing subjects, however, makes it of little value to the established scientist, and its non-clinical bias makes it of no help to the clinician.-RWF 